\title{
Suppression of stochastic pulsation in laser-plasma interaction by smoothing methods*
}

\author{
By M. AYDIN† AND H. HORA \\ †Department of Theoretical Physics, University of New South Wales, Kensington 2033, \\ Australia, and $\ddagger$ CERN, CH1211 Geneva 23, Switzerland
}

(Received 20 April 1992; accepted 8 July 1992)

Smoothing of laser-plasma interaction by ISI, RPP, SSD, etc. was mainly directed to overcome lateral nonuniformity of irradiation. While these problems are in no way less important, we derived numerically the model of the Laue rippling and hydrorelaxation model for explanation of the measured temporal pulsation in the 10- to 40-ps range and how the smoothing schemes suppress these pulsations. The partial standing wave fields of the normally coherent laser-irradiated plasma corona is then suppressed by smoothing and conclusion for tests for this model, e.g., by the "question mark experiment" is given. The result provides a physics solution of the laser interaction problem for direct-drive inertial fusion energy.

\section{Introduction}

After the last 30 years of extensive studies of laser-plasma interaction for the aim of laser fusion, it seems that one essential and dominating mechanism has been overlooked. The main emphasis was the study of instabilities and processes to avoid filamentation due to the lateral nonuniformity of the laser beams. It had not been realized that one basic difficulty is the temporal stochastic pulsation of the interaction with about 20-ps duration. Indications of this phenomenon were seen since 1973 and it may be clear finally that these complications are now overcome by smoothing, but smoothing - although experimentally successful - is interpreted and understood in a different way; that discrepancy may now be solved in view of the pulsation and its suppression.

Twenty years ago, it was known that the interaction of sufficiently high-intensity lasers with targets produced on the one hand purely thermal plasmas and on the other groups of plasmas with mostly superlinear and suprathermal properties. From laser-irradiated aluminum spheres, one could immediately see the thermally expanding core and the nonlinear cloud with $\mathrm{keV}$ ions (Engelhardt et al. 1970). The confusion about high- and lowtemperature X-ray signals was clarified for the first time by Eidmann (Büchl et al. 1972) showing that there are always both temperatures, a thermal one and a suprathermal temperature [due to "hot(?)" electrons]. When these plasmas were to be used of laser-driven fusion, a most difficult situation appeared with highly varying reflectivity, low transfer of energy into the plasma, hot spots, self-focusing, instabilities, anomalous kinds of scattering etc. The situation was as crazy as it is with today's impossibility to understand what is going on in a tokamak. One aim was to achieve a smooth interaction. The first ingenious solution was to use indirect drive (Nuckolls 1982), where one option is the cannonball, about which the first publication was by Yabe and Nishihara (1975), converting the laser radiation into $\mathrm{X}$ rays and letting these then compress and heat a fusion fuel pellet.

*Dedicated to Prof. Arthur Scharmann on his 65th birthday.

(C) 1993 Cambridge University Press $0263-0346 / 93 \$ 5.00+.00$ 
The nonsmooth interaction was considered to be caused by the nonuniform irradiation and smoothing was motivated by trying to spoil the otherwise ideal properties of the laser beam by artificially destroying the small spectral line width. When wide-band irradiation is produced (Deng et al. 1983, 1986) where the most sophisticated method is the induced spatial incoherence (ISI) (Lehmberg \& Obenschain 1983; Lehmberg et al. 1987), a temporal coherence of 1 ps only is achieved. Another way of smoothing was introduced by varying the direction of beamlets within the beam by using the random-phase plate (RPP) (Mima \& Kato 1982; Kato et al. 1984) or the "fly eye" lense array (Deng et al. 1983, 1986) or to combine diffraction and interference as "smoothing by spectral dispersion" (SSD) (Skupsky et al. 1989).

It seems evident that the motivation was mostly directed to suppress lateral nonuniformity of the laser intensity (Emery et al. 1991) when this type of smoothing was introduced. While these techniques proved an enormous success, e.g., the suppression of stimulated Raman scattering (SRS) from a few percent to hundred times lower values (Obenschain et al. 1989), it seems that their essential influence had not at all been understood. The understanding of lateral uniformity in the laser intensity is indeed an important aim and its study (Emery et al. 1991) is at least a point of partial understanding.

The new message in understanding the recent progress in laser interaction with plasmas by smoothing, however, may be of a basically different kind than that earlier discussed (Emery et al. 1991): lateral beam uniformity, namely, the most complex temporal pulsation of interaction with irregular sequences in the 10- to 40-ps range. A special view in this direction is presented here and a detailed numerical analysis may permit an understanding and an explanation of why most conditions of smoothing are just the suppression of this pulsation apart from the nonuniformity problem.

\section{Pulsation in laser-plasma interaction}

We mentioned the difficulty in lateral nonuniformity of irradiation in laser driving of plasmas. An obviously much more dangerous property is that of a strong pulsation of the interaction with a nonperiodic sequence in the 20-ps range, even if the incident laser intensity is completely constant in time. The first indication appeared from numerical studies. The Kinsinger code at ILE Rochester in 1973 described the 1-D interaction of a plane laser wave perpendicularly incident on a collisional plasma with some ramp of density profile.

Using the correct nonlinear optical constants with respect to the intensity dependence of the collision frequency and the ponderomotive and nonponderomotive terms of the nonlinear force, the two-temperature model showed a realistic response of the plasma [see figure 7.7a of Hora (1975) or figure 10.10a of Hora (1991a)]. First, the light penetrated to the critical density, from which point on the light decayed exponentially on the depth as expected and a strong mirror reflection produced a partial standing wave pattern with swelling and wavelength stretching as known from the nonlinear force. The absorption caused that the standing wave was partial only. At steplike switching on and off the laser, within less than $1 \mathrm{ps,} \mathrm{the} \mathrm{laser} \mathrm{pulse} \mathrm{did} \mathrm{no} \mathrm{longer} \mathrm{penetrate} \mathrm{to} \mathrm{the} \mathrm{critical} \mathrm{density} \mathrm{but} \mathrm{decayed}$ to $0.1 \%$ at the critical density; the light was then reflected at the low plasma density by the Bragg-von-Laue grating of the plasma density ripple that was produced by the nonlinear force in the standing waves.

While the net reflectivity was rather low when the light had a mirror reflection at the critical density due to the absorption of the light when moving back and forth in the plasma corona, the reflectivity was high at the phase reflection of the Laue grating. This was a downcasting result for laser fusion: The plasma did everything to prevent the light from being absorbed in the plasma. Motivated by this, Lubin and colleagues measured the reflec- 
tivity of a plasma when irradiated by a 100-ps laser pulse with nearly ideal rectangular time dependence and a rise and decay time of about 3-5 ps. The reflectivity showed then pulsation, changing between nearly $100 \%$ and a few percent within 10-20 ps irregularly up and down (Lubin 1974). The reflected $3 / 2$ harmonics had a similar pulsation but were out of phase with the reflectivity (Jackel et al. 1976), showing that only at low reflectivity the light went to the critical density where SRS produced the harmonics and was cut off when the reflectivity occurred at the low plasma density by phase reflection.

In recent years, similar pulsation or stuttering was observed. After discovering a completely irregular modulation of the backscattered spectra of the fundamental and the second harmonics of about $4 \AA$ width, it was clarified (Maddever 1988; Maddever et al. 1990) that an otherwise smooth laser pulse pulls the plasma corona to a velocity of some $10^{7} \mathrm{~cm} / \mathrm{s}$ within 10 (or so) ps and then the acceleration stops. After about 20-30 ps, another push is given to an additional velocity of the same kind. This gives just the modulated spectra while time-resolved detection is unmodulated but just showing the shifts (Maddever 1988; Maddever et al. 1990). A pulsation of the reflectivity between a few percent and $100 \%$ with an irregular sequence of about 20 ps was detected (Maddever 1988; Maddever et al. 1990), which was similar to Lubin's result (1974), and a likewise pulsation was measured for the $\mathrm{H}-\alpha \mathrm{X}$-ray emission (Rode et al. 1991). It was possible to clarify experimentally that the low reflection during the pulsation comes from the cut-off density while the high reflection is from the low-density outermost corona (Maddever 1988; Maddever et al. 1990).

Another pulsation in the 20-ps range was detected for the $3 / 2$ harmonics (Giulietti et al. 1989 ) and the most important thing is that when adding an RPP (Mima \& Kato 1982; Kato et al. 1984) for smoothing interaction the harmonics emission is completely smooth without pulsation. The 20-ps pulsation process was directly identified also from measurement of generation of $25-\mathrm{keV}$ ions of ionization up to $Z=18$ from tantalum targets irradiated by 30 ps or 3 ns neodymium glass laser pulses (Hora 1991b).

\section{Numerical analysis of the stuttering process}

Various models were discussed for explaining the mentioned pulsation. Motivated by the problems of lateral nonuniformity in connection with the RPP smoothing, filamentation and self-focusing was considered the reason for the pulsating $3 / 2$ harmonics, which simply are avoided by the RPP (Giulietti et al. 1989). Further, instabilities were discussed in this connection (Maddever 1988; Maddever et al. 1990). The fact that the pulsation is a rather stuttering mechanism with irregular random sequences induced the view of chaos (Guskov et al. 1991). While all these and other mechanisms may be involved partially or cannot be excluded easily, the following numerical study may provide an explanation for the dominating mechanism of pulsation or stuttering and a consequence for interpreting the smoothing by ISI, RPP, and other methods.

Knowing the interruption of the laser-plasma interaction as seen from the early computations [see the above-mentioned figures in Hora $(1975,1991 \mathrm{a})$ ] and the alternation from the mirror reflection at the critical density to the phase reflection by the Laue grating, we performed a computation with the real-time genuine two-fluid code of the laser-plasma interaction (Hora 1991a) for plane geometry including realistic nonlinear optical constants, nonlinear forces, equipartition time, and thermal conduction with time steps 10 times below the shortest plasma oscillation time and with Maxwellian exact numerical computations of the temporally and spatially changing laser field in the corona (Gu Min \& Hora 1991; Hora \& Aydin 1992; Aydin et al. 1992). Using a neodymium glass laser intensity of $10^{15} \mathrm{~W} / \mathrm{cm}^{2}$, the following initial conditions were chosen: electron and ion temperatures of $30 \mathrm{eV}$, zero 


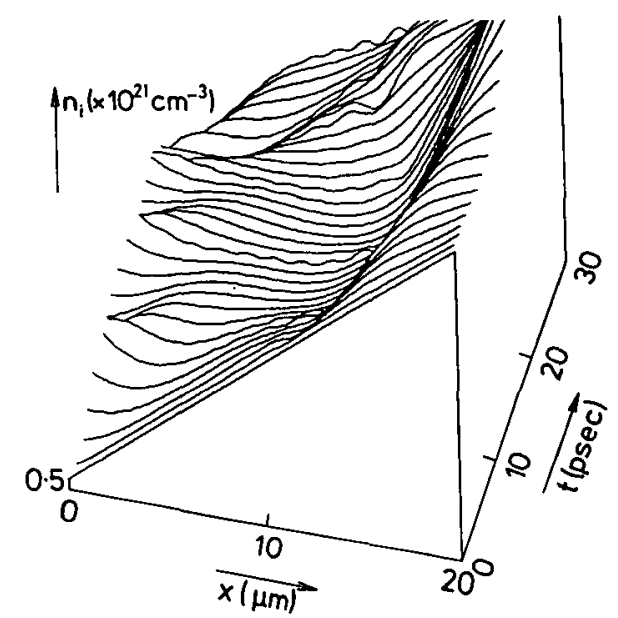

FIGURE 1. Time development of a $0.02-\mathrm{mm}$-thick plasma slab initially at rest and temperature of $30 \mathrm{eV}$ with increasing ion number density $\left(n_{i}\right)$ from $0.5-1.3$ times the critical density in a linear way, irradiated (from left-hand side) by a neodymium glass laser pulse of $10^{15} \mathrm{~W} / \mathrm{cm}^{2}$.

electron and ion velocities and a plasma of 20 wavelength thickness with a linear ramp of electron and ion densities growing from 0.5 at one side to 1.3 times the critical density at the other side; we see the following dependences up to $34 \mathrm{ps}$.

Figure 1 shows the ion density where the initially smooth slope is changed into ripples at about 5 ps. This is what happens about 1 ps later, when the electromagnetic laser field density shows the strong standing waves (figure 2). For times after $5 \mathrm{ps,}$, the laser field is nearly cut off at the low density (no standing wave field patterns) and the density ripples (figure 1) relax hydrodynamically to about $15 \mathrm{ps,} \mathrm{when} \mathrm{the} \mathrm{newly} \mathrm{penetrating} \mathrm{wave} \mathrm{field}$ again gets standing wave patterns (figure 2), producing density ripples, cutting off the penetration of light, etc. These ripples are synchronous with the ion velocities (figure 3), where on top it can be seen that the whole plasma corona receives a push during the first $5 \mathrm{ps,}$ the acceleration being then stopped and getting an additional push until 15 ps etc.

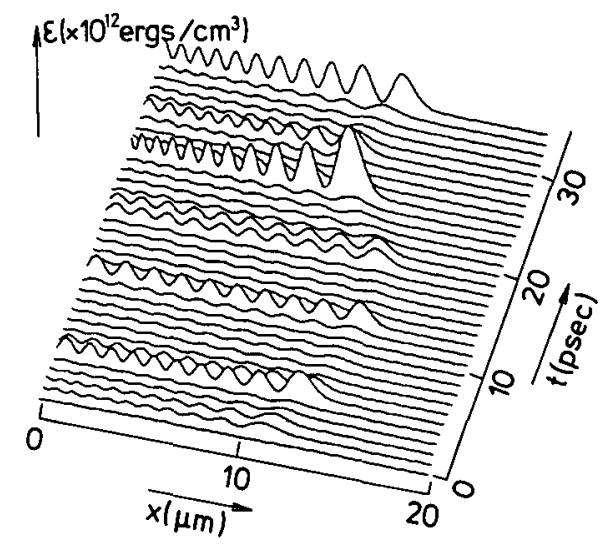

FIGURE 2. Time development of the electromagnetic energy density $(\varepsilon$ ) of the laser field for the same case as in figure 1 . 


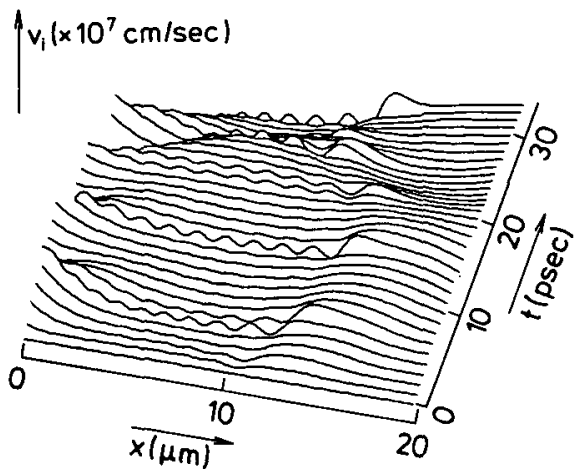

FigurE 3. Time development of the ion velocity $\left(v_{i}\right)$ of the irradiated plasma for the same case as in figure 1.

This all reproduces the observation of the stuttering interaction:

- the change from mirror reflection at the critical density (at about $14 \mu \mathrm{m}$ while dynamically changing in due course);

- the pulsation of reflectivity; and

- the sequence of pushes of the acceleration.

We can then explain the action of induced spatial incoherence (ISI) due to its temporal incoherence of 1 ps. In the computations, we have completely coherent laser waves. If the coherence changes within 1-3 ps, any standing wave field phases will change and will not permit partial standing waves or the subsequent density ripple or pulsation. We followed this up by the numerical case of a broad-band irradiation (Gu Min \& Hora 1991; Aydin et al. 1992; Hora \& Aydin 1992). With one single frequency, the time-dependent ripples appeared, while with a $1 \%$ frequency bandwidth laser pulse no pulsation or ripples appeared, but a nearly independent density and velocity field of up to $50 \mathrm{ps}$ was the result. Figures 4 and 5 show plots of numerical results for these two cases (with one single frequency and with three frequencies).

The action of the RPP (Mima \& Kato 1982; Kato et al. 1984) of the smoothing by spectral conversion (SSD) (Skupsky et al. 1989) or by the fly eye (Deng et al. 1983, 1986) may consist then in the generation of a highly complicated interference and diffraction field, where again the standing wave structure and the subsequent density rippling by the nonlinear force would be avoided.

It should be mentioned that attempts were made to relate broad-band irradiation with a decrease of parametric instability processes (Thomson \& Karush 1974; Weihan et al. 1992). This question is indeed important and motivated the use of broad-band irradiation experiments for smoothing (Deng et al. 1983, 1986). Because the action of instabilities does not dominate the plasma dynamics (Labaune et al. 1985; Drake 1988; Hora 1991a), its involvement with the laser-plasma interaction seems to have lower priority compared with the stuttering mechanism.

\section{Consequences of the Laue rippling and hydrorelaxation model}

It should be underlined that the just-developed model of the stuttering only covers the 1-D development. Any lateral nonuniformity discussion will have to be added (Emery et al. 1991) and there will be a rich source for studying chaos (Guskov et al. 1991). Whether 


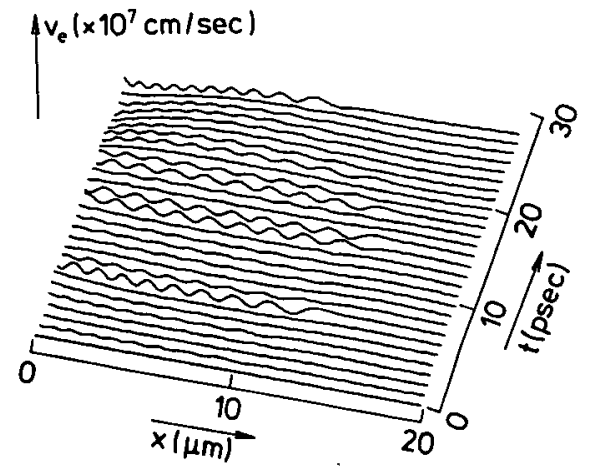

(a)

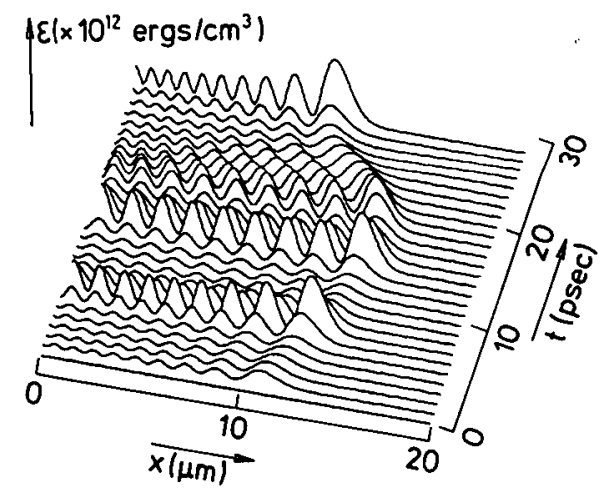

(c)

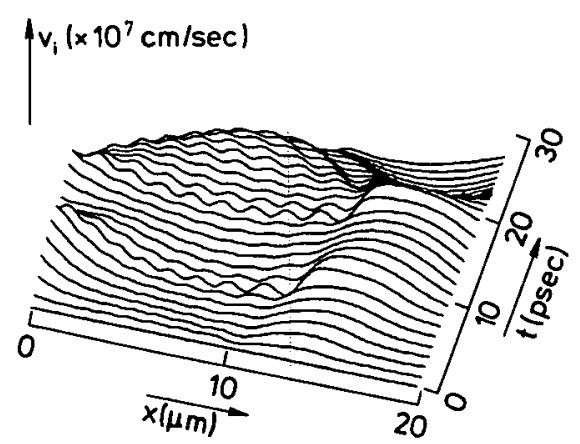

(b)

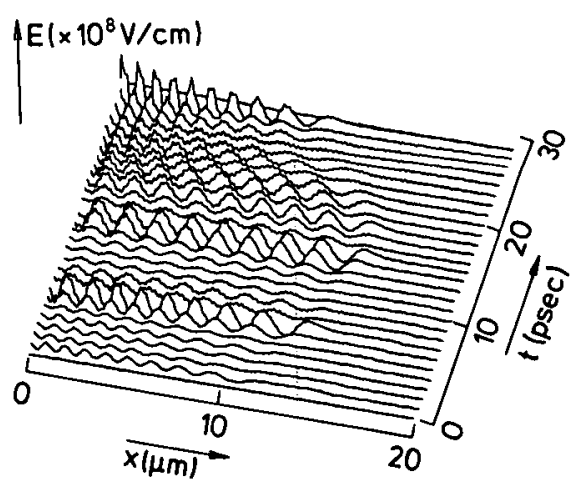

(d)

FIGURE 4. (a) Electron velocity $\left(v_{e}\right)$ profiles similar to the case of figure 1 but for 0.6 times the laser intensity when irradiated by one single laser frequency with a pulsating generation of ripples. (b) Ion velocity $\left(v_{i}\right)$ profiles for the same case as in (a). (c) Electromagnetic energy density ( $(\varepsilon)$ for the same case as in (a). (d) Longitudinal electric field $(E)$ for the same case as in (a) ( $E$ is not filtered).

our mentioned model of Laue rippling with hydrorelaxation and the conclusion for avoiding this all by smoothing mechanisms in the explained way is the dominating process may be seen from the following conclusions.

The appearance of the pulsation may have been connected with the question mark experiment (Eidmann \& Sigel 1974; Sigel et al. 1976). It was observed that the reflection is a phase reflection and not a mirror reflection, by which way a question mark sign is turned upside down. If this experiment will be repeated, it is expected on the basis of our model that the question mark will change in a pulsating way within 20-30 ps from a weak and short, normal upright mirror reflection to strong, down-turned phase reflection back and forth. Using the right smoothing, e.g., ISI, the question mark should be mirror reflected, weak, upright, and nearly temporally unchanged in intensity.

In another experiment, one may confirm that the pulsation of reflectivity (Maddever 1988; Maddever et al. 1990) of the $\mathrm{H}-\alpha$ emission (Rode et al. 1991) should be suppressed by using an RPP. This smoothing should again result in a modest reflection.

If it is then clarified that the smoothing - apart from lateral improvements of uniformity of irradiation - overcomes the pulsation and provides low reflectivity and results in nearly constant high deposition of laser energy into the plasma corona, then the conditions of direct-drive laser fusion will be achieved ideally. This is then the physics solution (Hora 

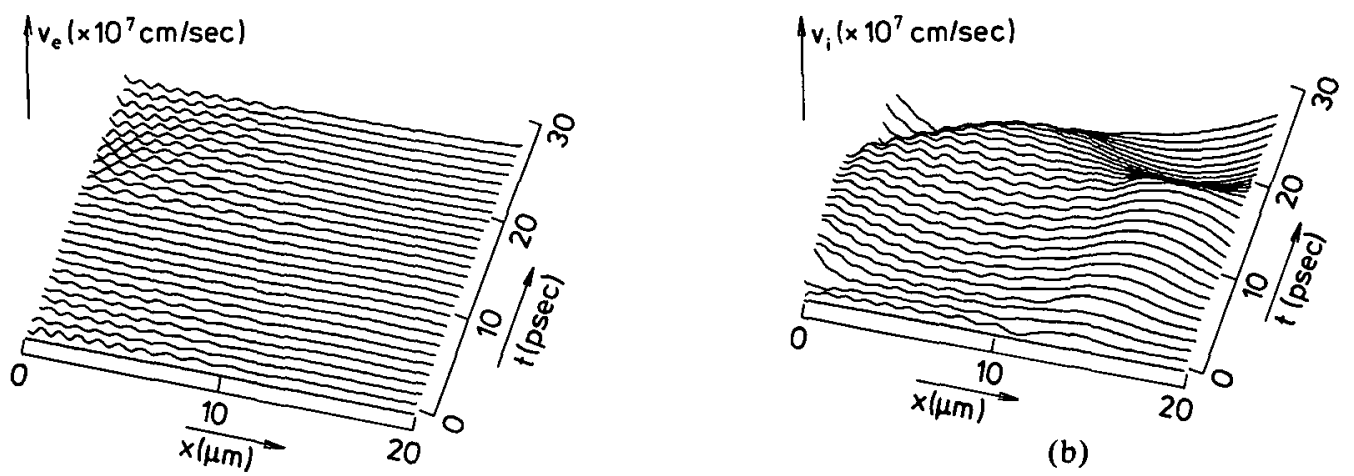

(a)

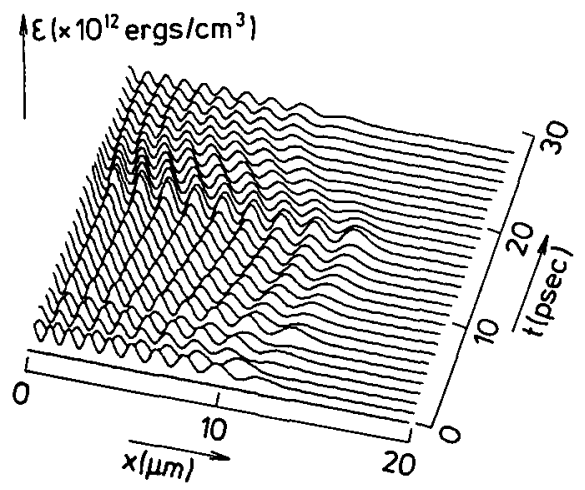

(c)

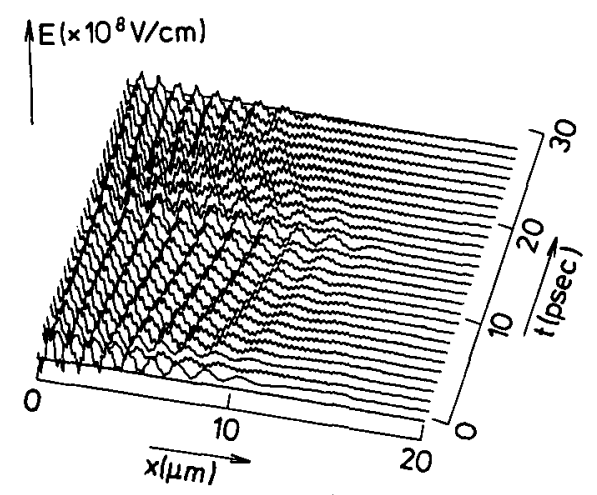

(d)

FIGURE 5. (a) Same as figure 4(a) with irradiation of a spectrum of $1 \%$ spectral width by three laser frequencies $0.5 \%$ spectrally apart. (b) Same as figure 4(b) with irradiation of a spectrum of $1 \%$ spectral width by three laser frequencies $0.5 \%$ spectrally apart. (c) Same as figure 4(c) with irradiation of a spectrum of $1 \%$ spectral width by three laser frequencies $0.5 \%$ spectrally apart. (d) Same as figure 4(d) with irradiation of a spectrum of $1 \%$ spectral width by three laser frequencies $0.5 \%$ spectrally apart.

1991a, 1992) - together with the reliable and not parameter-dependent scheme of volume ignition (Eliezer \& Hora 1993; Pierushka et al. 1992; Khoda-Bakhsh 1993; Stening 1993)for the early available and low-cost clean energy production by laser fusion.

\section{REFERENCES}

AYDIN, M. et al. 1992 Laser Particle Beams 10, 155.

BüchL, K. et al. 1972 In Laser Interaction and Related Plasma Phenomena, H. Schwarz et al., eds.

(Plenum, New York), Vol. 2, p. 503.

Deng, X. et al. 1983 Acta Optica Sinica 2, 97.

Deng, X. et al. 1986 Appl. Opt. 25, 377.

Drake, R.P. 1988 Laser Particle Beams 6, 235.

Eidmann, K. \& Sigel, R. 1974 In Laser Interaction and Related Plasma Phenomena, H. Schwarz et al., eds. (Plenum, New York), Vol. 3B, p. 667.

ElIEZER, S. \& HORA, H. 1991 In Inertial Fusion by Inertial Confinement, G. Velarde, Y. Ronen, and J.M. Martinez-Val eds. (CRC Press, Boca Raton) p. 43.

EMERY, M.H. et al. 1991 Phys. Fluids B 3, 2640. 
Engelhardt, A.G. et al. 1970 Phys. Fluids 13, 212.

Gu M. \& Hora, H. 1991 Laser Particle Beams 9, 381.

Giuletti, A. et al. 1989 In Laser Interaction with Plasmas, G. Velarde et al., eds. (World Scientific, Singapore), p. 208.

Guskov, S. et al. 1991 ECLIM'91 Warsaw Conference paper P-66.

HorA, H. 1975 Laser Plasmas and Nuclear Energy (Plenum, New York).

Hora, H. 1991a Plasmas at High Temperature and Density (Springer, Heidelberg).

HORA, H. et al. 1991b CERN-PS Report.

HoRA, H. 1992 Encyclopedia of Physical Science and Technology (Academic Press, New York) 2nd ed., Vol. 8, p. 433.

Hora, H. \& Aydin, M. 1992 Phys. Rev. A 45, 6123.

JACKel, S. et al. 1976 Phys. Rev. Lett. 37, 95.

Kato, Y. et al. 1984 Phys. Rev. Lett. 53, 1057.

KhODA-BaKhSH, R. 1993 Nucl. Instrum. Meth. (in press).

Labaune, C. et al. 1985 Phys. Rev. A. 32, 577.

Lehmberg, R.H. \& Obenschain, S.P. 1983 Opt. Comm. 46, 27.

Lehmberg, R.H. et al. $1987 \mathrm{~J}$. Appl. Phys. 62, 2680.

LuBIN, M. 1974 IN ECLIM'74 Garching Abstracts, p. 34.

MaddeVER, R.A.M. 1988 Ph.D. thesis, Australian National University.

MAdDever, R.A.M. et al. 1990 Phys. Rev. A. 41, 2154.

Miмa, K. \& Kaтo, Y. 1982 Institute of Laser Engineering, Osaka University Progress Report on Inertial Confinement Fusion.

Nuckolls, J.H. 1982 Phys. Today 35 (9), 24.

ObensChain, S.P. et al. 1989 Phys. Rev. Lett. 62, 768.

PIERUSHKA, P. et al. 1992 Laser and Particle Beams 10, 145.

SigeL, R. et al. 1976 Phys. Rev. Lett. 36, 1369.

Skupsky, S. et al. $1989 \mathrm{~J}$. Appl. Phys. 66, 3456.

Stening, R.J. et al. 1993 In Laser Interaction and Related Plasma Phenomena, G.H. Miley et al. eds. (Plenum, New York) Vol. 10, p. 493.

Thomson, J.J. \& Karush, J.I. 1974 Phys. Fluids 17, 1607.

WeIHAN, T. et al. 1992 Laser Particle Beams 10, 1.

YABE, T. \& NishiHARA, K. 1975 Research Report IPP-J-235, Nagoya University. 